\title{
Comparison of the efficacy and safety of circumcision by freehand technique and Plastibell device in children
}

\author{
Babatunde K. Hamza ${ }^{1,2^{*}}$ (D) Muhammed Ahmed ${ }^{3}$, Ahmad Bello ${ }^{3}$, Tunde Talib Sholadoye ${ }^{3}$, \\ Musliu Adetola Tolani ${ }^{3}$, Ahmad Tijjani Lawal ${ }^{3}$, Muhammad Salihu Muhammad ${ }^{2}$, Khalifah Ibrahim Abdulsalam², \\ Nasir Oyelowo ${ }^{3}$ and Hussein Yusuf Maitama ${ }^{3}$
}

\begin{abstract}
Background: Circumcision has been practiced since antiquity and is one of the most commonly performed surgical procedures. The aim of the study was to compare the efficacy and safety of the two most common techniques: Freehand and Plastibell techniques of circumcision.

Methods: The study was a prospective randomized study that was conducted on uncircumcised males at or below the age of 5 years that presented for circumcision. All boys who satisfied the inclusion criteria and whose parents consented, were randomized into two groups. Group A had freehand circumcision, while Group B had Plastibell circumcision. Patients were followed up at day 7 and day 28 postoperatively. The variables compared were operating time, cost, complications and parents'satisfaction. All the data obtained were entered into a proforma. The data obtained were analyzed using SPSS version 20.

Results: A total of 110 boys were circumcised, 55 boys in each group. The median ages in the Plastibell and freehand groups were one and three months respectively. The mean operating time was significantly shorter in the Plastibell group compared to freehand (3.53 min versus $16.7 \mathrm{~min}$ ). Total cost implication per procedure was also cheaper in Plastibell compared to freehand (3700 versus 6600). However, complications were more in the Plastibell group compared to freehand $(29.1 \%$ versus $9.1 \% ; \mathrm{P}=0.008)$. These complications in Plastibell group were age related. Parents in both groups were equally satisfied with the outlook on day 28.
\end{abstract}

Conclusion: Plastibell circumcision has the advantage of being faster and cheaper than freehand circumcision. Complications were more in the Plastibell group, and they were found to be age related.

Keywords: Circumcision, Freehand, Plastibell

\section{Background}

Circumcision is arguably the oldest surgical procedure [1]. Religious, cultural, medical and recently public health reasons are indications for the procedure [2]. Religious and or cultural circumcision is practiced by Christians, Jews, Muslims, Black Africans, Australian aborigines and

\footnotetext{
${ }^{*}$ Correspondence: ridwankolapo@yahoo.com

${ }^{1}$ Department of Surgery, Kaduna State University, Kaduna, Nigeria

Full list of author information is available at the end of the article
}

other ethnic groups in different parts of the world. Male circumcision is an important surgical procedure of public health importance as it is associated with some diseases and complications.

Although many techniques of circumcision have been described, there are few reports determining which technique is associated with the least complications [3]. Conventional dissection surgery (freehand) or Plastibell technique is the most frequently employed method for circumcision [4]. 
The aim of this study is to compare the efficacy and safety of freehand and Plastibell techniques of circumcision.

\section{Methods}

The study was a prospective study conducted over a period of 1 year, between November 2016 and October 2017 , on boys $\leq 5$ years, who presented for circumcision at the surgical outpatient department, of our hospital. Approval was obtained from the health research ethics committee (HREC) of our hospital (with reference number-HREC/CL/05) prior to the commencement of the study. A written informed consent was obtained from all the parents for participation in the study after adequate counseling. Patients with hypospadias, epispadias, micropenis, disorders of sexual development, micropenis and infective conditions of the external genitalia were excluded.

The patients were randomized assigned into two groups. The samples were allocated into two groups by a simple randomization technique using computer generated random numbers. The choice of procedure was determined based on whether the number falls into either group A or B. Group A had freehand circumcision, while Group B had Plastibell circumcision. The cost implication per patient was noted. All the surgeries were performed by the same surgeon.

The primary outcome was to compare the complication rate and parental satisfaction, while the secondary outcome was to compare operating time and cost.

\subsection{Anesthesia}

Local anesthesia was used for patients that are 1 year and below. Plain $0.5 \%$ lidocaine was used at a dose of $3 \mathrm{mg}$ per kilogram body weight. The local anesthetic was injected with a $25 \mathrm{G}$ needle. The dorsal penile nerves which were located at the 11 and 1 O'clock positions were blocked. This was followed by a circumferential ring block. Five minutes was allowed to elapse before commencing the circumcision procedure.

General anesthesia was used for boys greater than 1 year.

\subsection{Procedure}

In the freehand technique, the tip of a curved mosquito artery forceps was inserted into the preputial opening following which the artery forceps was opened and used to dilate the prepuce. The prepuce was later retracted. Adhesion between the prepuce and the glans was separated gently until the coronal sulcus was well demarcated. The prepuce was then returned to its normal position. A straight mosquito artery forceps was used to crush the prepuce at 12 O'clock position for one minute. The clamp was opened and removed; then, two curved hemostatic clamps were applied at the 10 and 2 O'clock positions of the prepuce. With the aid of scissors, a cut is made at 12 O'clock position through the crushed skin to the level of the corona. The prepuce was cut all around, taking care to leave about $5 \mathrm{~mm}$ of the inner leaf of preputial skin. Any significant bleeding was controlled by clipping the blood vessel with an artery forceps and then ligated. The inner and outer preputial skins were sutured together with vicryl 4-0 using interrupted technique. Wound dressing was applied using Sofra-Tulle and sterile gauze.

For performing a Plastibell circumcision, the tip of a curved mosquito artery forceps was inserted into the preputial opening following which the artery forceps was opened and used to dilate the prepuce. The prepuce was later retracted. Adhesion between the prepuce and the glans was separated until the coronal sulcus was well demarcated. The prepuce was then returned to its normal position. A small dorsal slit was made at 12 O'clock position of the prepuce, just wide enough to admit the Plastibell ring. The bell of the Plastibell device was inserted over the glans (the appropriate size should cover two-third of the head of the glans). Then the prepuce is tied all around, over the groove of the bell, with a tight ligature (which usually comes with the device). The prepuce distal to the string was cut off. No wound dressing was applied.

Operating time was timed with the same digital stopwatch by a house officer who did not participate at the surgery. Operating time in the freehand technique was from the first clamp applied at the 12 O'clock position to the last stitch, while the operating time in the Plastibell was from the start of the dorsal slit incision to the excision of the redundant prepuce.

The procedures were done as day cases. The parents were asked to come with the circumcised child for follow-up on days 7 and 28.

The plastic ring was allowed to separate within 14 days. Any ring that failed to separate after 14 days was tagged as delayed separation and was manually removed.

Parental satisfaction was assessed based on the appearance of the penis on day 28 . This was scored as either satisfactory or unsatisfactory.

All the data obtained were entered into a proforma. The data obtained were analyzed using SPSS version 20 . A confidence level of $95 \%$ was used, and $p$ value $\leq 0.05$ was considered to be significant. Data were expressed as means \pm standard deviation, medians, absolute values and percentages. Student's t test was used to compare continuous variables. The frequency of complications between the two groups was compared using Chi-square test. 


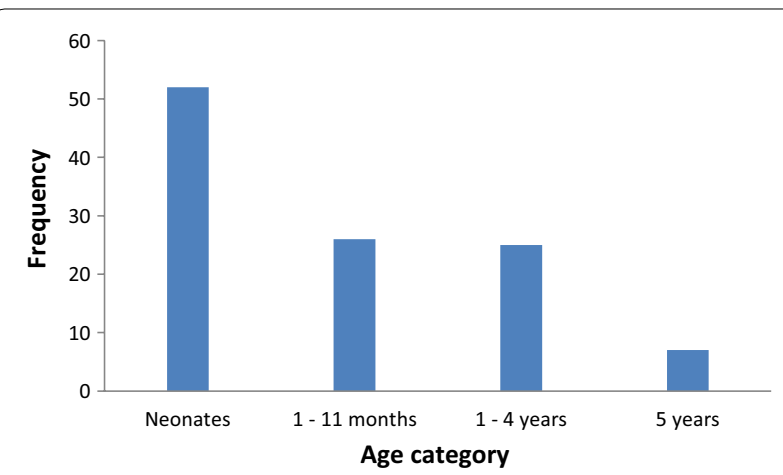

Fig. 1 Age categories of all circumcised boys

\section{Results}

A total of 120 boys were circumcised during the study period. Ten boys were excluded due to inadequate data and loss to follow-up. Complete data were obtained for 110 boys, 55 in each group, and were subjected to analysis. The age categories of all boys circumcised are displayed in Fig. 1.

The predominant tribe was Hausa, which constituted $87.2 \%$ (96 boys). Ten percent (11 boys) were Yorubas and $2.7 \%$ (three boys) were Ibos. Majority of the parents were Muslims (91.8\%), and the remaining 8.2\% were Christians. Religion was overwhelmingly the indication for circumcision which was found in $94.5 \%$ (105 boys), while $5.5 \%$ (5 boys) were circumcised for cultural reasons.

The median age in the freehand was 3 months (range of 8 days -5 years), while the median age in the Plastibell group was 1 month (range of 9 days -5 years). The median age difference was not statistically significant $(P=0.18)$. Table 1 shows a comparison of the age categories in the two groups.

The mean operating time in the Plastibell group was $3.53 \pm 1.21 \mathrm{~min}$ with a range of $2-9 \mathrm{~min}$. The mean operating time in the freehand group was $16.7 \pm 2.67 \mathrm{~min}$ with a range of $10-25 \mathrm{~min}$. The operating time in the Plastibell group was found to be shorter than the freehand group. This was found to be statistically significant $(P<0.001)$.

The average cost of per patient (operation fee, consumables, sterilization) was $\$ 6600$ for freehand circumcision and $\$ 3700$ for Plastibell circumcision (Table 2).

Superficial surgical site infection (evidenced by the presence of pus) was diagnosed on day 7 in four boys $(7.3 \%)$ in the freehand group versus three boys $(5.5 \%)$ in the Plastibell group $(P=1.00)$. This was not statistically significant. In the Plastibell group, two boys still had their plastic rings in place when they developed surgical site infection. The plastic rings were manually removed (in a 2-week-old and a 2-month-old on day 3 and day 7 ,
Table $1 \mathrm{~A}$ table comparing the age categories of the freehand and Plastibell circumcision groups

\begin{tabular}{llll}
\hline Age categories & Freehand & Plastibell & Total \\
\hline Neonates & 23 & 29 & 52 \\
$1-11$ months & 14 & 12 & 26 \\
$1-4$ years & 13 & 12 & 25 \\
5 years & 5 & 2 & 7 \\
Total & 55 & 55 & 110 \\
\hline
\end{tabular}

Table 2 A table comparing the total cost implication per procedure

\begin{tabular}{lll}
\hline & Freehand & Plastibell \\
\hline Surgical fee & $\# 5,000$ & $\# 3,000$ \\
Cost of consumables & $\# 1,300$ & $\# 400$ \\
Sterilization & $\# 300$ & $\# 300$ \\
Total & $\# 6,600$ & $\# 3,700$ \\
\hline
\end{tabular}

respectively). The ring had already fallen off in the third patient. They all did well on oral antibiotics and daily sitz bath.

Five patients $(9.1 \%)$ had bleeding complications in the Plastibell group (see Fig. 2). Their age range was 5-48 months. A five-month-old infant re-presented after he had left the hospital. Two boys developed rapidly increasing distal penile swelling secondary to hematoma, just proximal to the ligature. These were noticed on the operating table. The ligatures were released, and the hematoma was evacuated. The remaining two boys were noticed to be bleeding overtly

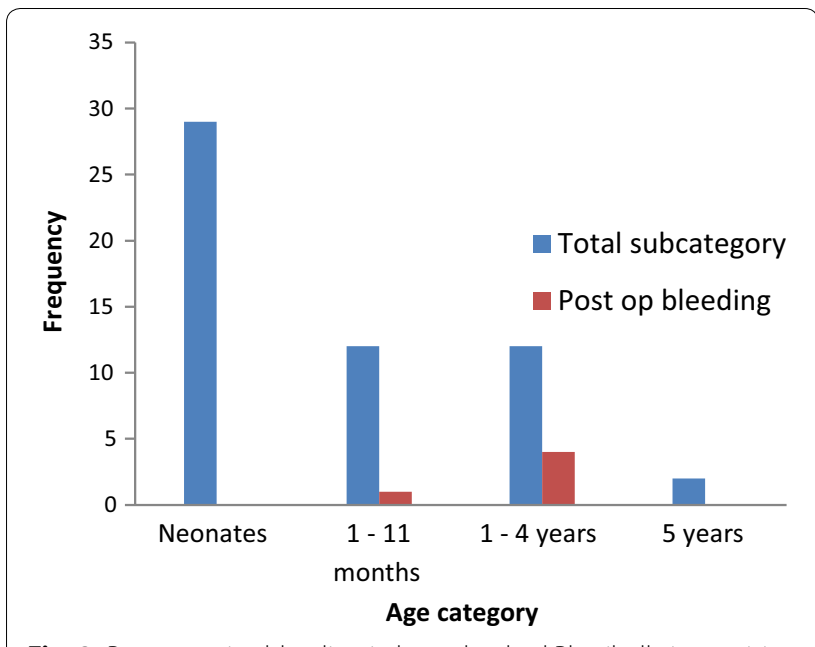

Fig. 2 Postoperative bleeding in boys that had Plastibell circumcision 


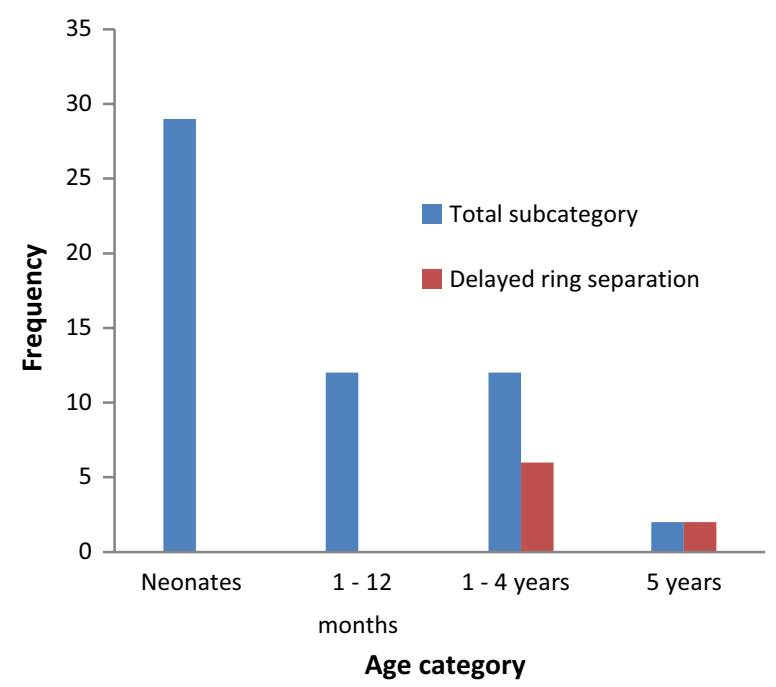

Fig. 3 Boys that had delayed separation of the Residual plastic ring following Plastibell circumcision

within the first one hour of the surgery, while in the recovery room. All had conversion to freehand technique with ligation of the bleeding vessels. No postoperative bleeding occurred in the freehand group. Comparison of bleeding complications between the two groups was not statistically significant $(P=0.057)$.

One neonate in the freehand group had adhesion at 1 month. The skin of the distal penal shaft was adherent to the corona. This was separated via blunt dissection. No adhesion occurred in those that had Plastibell circumcision.

Eight boys had delayed separation of the Plastibell ring (the ring had not fallen by day 14). Their age range was $2-5$ years (see Fig. 3 ). All the patients with delayed separation of the ring had a second procedure for the removal of the retained Plastibell ring. They all did well subsequently.

The Plastibell ring fell most commonly on day 6 for those that had spontaneous separation, with a range of 2-11 days. It separated faster in neonates compared to other age-groups. In neonates, the mean day the ring fell was $5.68 \pm 1.96$ days, while in age-group 1-11 months, the mean day was $7.09 \pm 2.55$ days. In the age-group 1-4 years, the mean day was $10.5 \pm 0.7$ days. The ring never fell within 14 day in those that were five years old. After the 11th day, there was no ring that separated spontaneously until the 14th day when they were extracted (see Fig. 4).

The Plastibell ring comes in several sizes (1.1$1.7 \mathrm{~cm}$ ). The size that was used most commonly was $1.3 \mathrm{~cm}$. The frequency with which each size was used is represented in Fig. 5.

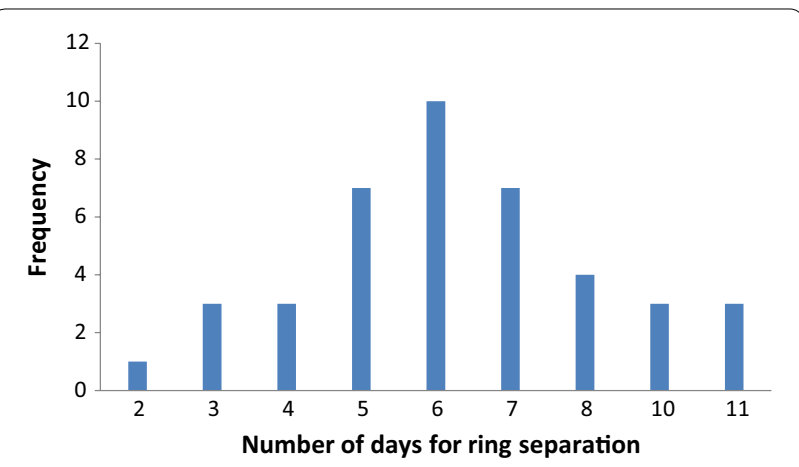

Fig. 4 Number of days for ring separation following Plastibell circumcision

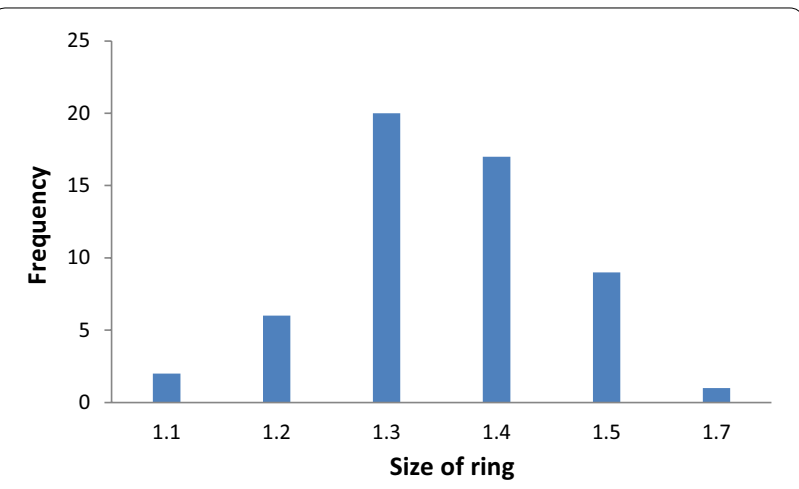

Fig. 5 Plastibell sizes (cm) used in circumcision

Table 3 Comparison of complications of freehand and Plastibell circumcision among 110 boy $\leq 5$ years

\begin{tabular}{llll}
\hline & Freehand & Plastibell & $\boldsymbol{p}$ value \\
\hline Bleeding & 0 & 5 & 0.057 \\
Surgical site infection & 4 & 3 & 1.00 \\
Adhesion & 1 & 0 & 1.00 \\
Delayed separation & - & 8 & \\
Complication rate & $9.1 \%$ & $29.1 \%$ & 0.008 \\
\hline
\end{tabular}

There was no correlation between the size of the ring and number of days for separation (Pearson's correlation coefficient $=0.266, P=0.122$ ). Age had a significant correlation with the number of days for separation (Pearson's correlation coefficient $=0.510, P<0.001$ ).

In the freehand group, three boys had prolonged penile swelling which persisted till day 7 . These swellings subsided before the next follow-up at 1 month. No patient in the Plastibell group had penile swelling on day 7 and beyond. 


\begin{tabular}{|c|c|c|}
\hline Grade & $\begin{array}{l}\text { Number } \\
\text { of patients }\end{array}$ & Complications \\
\hline$\|$ & 7 & Surgical site infection requiring antibiotic therapy \\
\hline Illa & $\begin{array}{l}1 \\
1\end{array}$ & $\begin{array}{l}\text { Adhesion-managed by blunt dissection } \\
\text { Reactionary hemorrhage from Plastibell requiring } \\
\text { conversion to freehand technique }\end{array}$ \\
\hline $111 \mathrm{~b}$ & $\begin{array}{l}4 \\
8\end{array}$ & $\begin{array}{l}\text { Reactionary hemorrhage from Plastibell requiring } \\
\text { conversion to freehand technique } \\
\text { Delayed Plastibell ring separation requiring } \\
\text { removal }\end{array}$ \\
\hline
\end{tabular}

Table 5 Comparison of complications in different age categories between freehand and Plastibell techniques

\begin{tabular}{llll}
\hline Age category & Freehand & Plastibell (\%) & $\boldsymbol{p}$ value \\
\hline Neonates & $4.3 \%$ & 3.4 & 1.000 \\
1-11 months & 0 & 25 & 0.085 \\
1-4 years & $15.4 \%$ & 83.3 & 0.001 \\
5 years & $40 \%$ & 100 & 0.429 \\
\hline
\end{tabular}

The overall complication rate was higher in the Plastibell group compared to freehand (29.1\% versus 9.1\%), as displayed in Table 3 . This was statistically significant $(P=0.008)$. The complications according to CavienDindo classification is displayed in Table 4.

In the freehand group, there was no correlation between age and frequency of complications (Pearson's correlation coefficient $=0.187, P=0.171$ ), while in the Plastibell group, there was a strong correlation between age and frequency of complications (Pearson's correlation coefficient $=0.752, P<0.001)($ see Table 5$)$.

All the parents in the two groups were satisfied with the cosmetic outlook at 1 month.

\section{Discussion}

Neonates were the commonest age-group that were circumcised in this study constituting $47.3 \%$. Older children (1-5 years), however, constituted a significant proportion $(29.1 \% \%)$ in this study due to cultural practice in northern Nigeria. All boys age-group 1-5 years recruited to this study were Hausas, who tend to circumcise children at older ages compared to other cultures. Studies conducted in other parts of the country reported younger ages for childhood circumcision. Bioku et al. [5] in a multicenter study involving centers in Lagos and Abuja reported an age range of 4 days to 3 months, and $61.2 \%$ were circumcised during the second week of life. They adduced the study findings to the settings (Abuja and Lagos) in which preponderance of the patients were Yorubas and Ibos, who typically circumcise their children earlier and often within the first few days of life. Ekwunife et al.[6] in a study conducted at Nnamdi Azikwe University Teaching Hospital, Nnewi (southeastern Nigeria), reported an age range of $2-140$ days, and $73.9 \%$ of the parents would prefer circumcision to be carried out on the 8th day of life.

It takes significantly more time to perform freehand circumcision as compared to Plastibell. This is similar to the findings of Neeto et al. [7], in a prospective randomized trial who reported $3.29 \mathrm{~min}$ for Plastibell versus $14.64 \mathrm{~min}$ in the dissection circumcision. Mousavi et al. [4] reported an average operating time of $3.4 \mathrm{~min}$ in Plastibell versus $9.2 \mathrm{~min}$ in conventional open surgery (sleeve resection). The operating time in the Mousavi's conventional open surgery is shorter than that reported by this study (9 versus $16.7 \mathrm{~min}$ ). This may be due to the fact that Mousavi's study population were infants, who tend to have less developed tissues and likely less intraoperative bleeding compared to older children. Mousavi had a postoperative bleeding complication rate of $1.95 \%$ ( 4 infants); meanwhile, there was no postoperative bleeding in the freehand group of this study, likely due to meticulous hemostasis. He also used a sleeve method which is different from the type of freehand employed in this study.

Size is paramount in Plastibell circumcision. The Plastibell comes in different sizes $(1.1-1.7 \mathrm{~cm})$. Appropriate size is determined by visual estimate of the glans girth which gets better with experience. The appropriate size should fit snugly over two-third of the glans [8]. This is important as oversize and undersize rings may cause proximal migration and tissue necrosis, respectively. The most common size of ring used in this study was size $1.3 \mathrm{~cm}$. Similar findings were reported in other series [5, 9].

The residual plastic ring usually falls within 7-10 days [10] In this study, the mean duration of separation of the plastic ring in days was 5.68, 7.09 and 10.5 in neonates, 1-11 months and age-group 1-4 years, respectively. The ring separated faster in neonates compared to all other age categories. This can be attributed to thinner prepuce.

Some series reported delayed separation of the ring as the commonest complication of Plastibell, which is commoner in older children $[4,11,12]$. This was also the commonest complication in this study which occurred in eight boys (14.5\%). This can also be attributed to substantial number of children 1-5 years in the Plastibell group (25.5\%). No neonate or infant had delayed separation.

In this study, 14 days was the maximum time allowed for separation of the residual plastic ring, although Neeto et al. [13] in a prospective study of Plastibell circumcision of older children (age range 5-13 years), reported spontaneous separation of the plastic ring even up to 26 days. 
However, such protocol is time-consuming and increases patient morbidity. The role of the plastic ring is to protect the glans during the circumcision; its continued retention is for hemostasis and ischemic necrosis of the preputial skin. These processes should be complete within $24 \mathrm{~h}$. Bode et al. [14] in a prospective study of penile injuries resulting from proximal migration of Plastibell ring at Lagos University Teaching Hospital, reported 23 injuries resulting from prolonged retention of the Plastibell. The authors suggested that it would be safest to remove the ring the following day after the procedure.

Five patients $(9.1 \%)$ in the Plastibell group had postoperative bleeding. Causes of bleeding during Plastibell include inappropriate size (too big a size) and inadequate tying of ligature around the device, predisposing to a displacement of the plastic ring with retraction of the inner prepuce, which leads to release of the compression over the preputial vessels and consequent hemorrhage. Two patients likely had retraction of the inner prepuce, while the ring was being applied. They developed distal penile swelling from hematoma after the ligature had been tied.

The bleeding rate in the Plastibell group was 9.1\% which is lower than that of Mousavi et al. [4] who reported a bleeding rate of $18 \%$ in a prospective study of infants $\leq 12$ months and also lower than that Lazarus et al. [15] who had a bleeding rate of $44 \%$ in a retrospective review. Palit et al. [16] had a lower bleeding complication rate of $3 \%$ compared to this study; however, their study population was younger, consisting of infants, ages between 6-14 weeks. All the patients that had postoperative bleeding in this study were older children (5-48 months). Bleeding seems to be more common in older children.

The incidence of surgical site infection was $5.5 \%$ in the Plastibell group versus $7.3 \%$ in the freehand group $(P=1.00)$. Circumcision being a clean surgery should have a lower rate of surgical site infection. A possible explanation of the infection rate may be due to the fact that the local wound care at home may be suboptimal and limited control of older children from activity and contamination. The infection rate was, however, found to be lower than Mak et al. [17] (13.7\% in Plastibell and $14.9 \%$ in dissection group), but higher than Fraser [18] (4\% with both techniques). Mousavi et al. [4] reported a very low rate of infection (1\% in Plastibell and none in freehand group). Mousavi attributed the likely reason to application of topical antibiotic ointment.

One neonate in the freehand group represented at 1 month with adhesion of the skin of the penile shaft to the corona. Even though this adhesion was released via blunt dissection, most postcircumcision adhesions require no treatment, they usually resolve with time. The chance of postcircumcision adhesion decreases with ageing [19]. Adhesions are generally commoner in freehand technique compared to Plastibell [13].

There were no major complications in this study. Complications of circumcision are very few in skilled hands [20]. There were minor complications which were easily managed. The overall complication rate was more in the Plastibell group compared to the freehand group (29.1\% versus 9.1\%; $P=0.008$ ). The complications in Plastibell were age related and commoner in older children.

\section{Conclusion}

Plastibell circumcision has the obvious advantage of being faster than freehand circumcision. The total cost implication per procedure is also far cheaper as compared to freehand technique. These are quite beneficial to high-volume centers. Plastibell has the best outcome in infants. Older children are at risk of bleeding and delayed separation of the residual plastic ring. Close follow-up should be enforced following Plastibell circumcision.

Freehand circumcision is safe for all groups, but it is more time-consuming. The complication rate is lower compared to Plastibell, particularly in older children.

\section{Acknowledgements \\ Not applicable}

\section{Authors' contributions}

BKH was involved in project development, manuscript writing, data collection, data analysis and editing. MA, AB and HYM took part in project development and editing. TTS, ATL, MSM, KIA and NO performed data collection and editing. MAT carried out data collection, data analysis and editing. All authors read and approved the final manuscript.

\section{Funding}

None.

Availability of data and materials

Raw data are available at the request of the author.

Ethics approval and consent to participate

This study was approved by the health research ethics committee (HREC) of Ahmadu Bello University Teaching Hospital Zaria (Reference number: ABUTH/ HREC/CL/05). A written informed consent was obtained from the parents of the patients before they were recruited into the study.

\section{Consent for publication}

Consent for publication was obtained from the parents of the participants.

\section{Competing interests}

None.

\section{Author details}

${ }^{1}$ Department of Surgery, Kaduna State University, Kaduna, Nigeria. ${ }^{2}$ Ahmadu Bello University Teaching Hospital, Zaria, Nigeria. ${ }^{3}$ Ahmadu Bello University/ Ahmadu Bello University Teaching Hospital, Zaria, Nigeria.

Received: 15 June 2020 Accepted: 16 September 2020 Published online: 16 November 2020 


\section{References}

1. Doyle D (2005) Ritual male circumcision: a brief history. J R Coll Phys Edinburg 35(3):279-285

2. Templeton DJ (2010) Male circumcision to reduce sexual transmission of HIV. Curr Opinion HIV/AIDS 5(4):344-349

3. Mak YLM (1995) Childhood circumcision: conventional dissection or plastibell device - a prospective randomized trial. Hong Kong Pract 17(3):101-105

4. Mousavi SA (2008) Circumcision complications associated with the plastibell device and conventional dissection surgery: a trial of 586 infants of ages up to 12 months. Adv Urol 10(6):1155-1160

5. Bioku MJ, Ikuerowo SO, Igwilo C, Omisanjo OA, Oluwafemi A, Olusanmi EJ (2016) Plastibell circumcision of 2,276 male infants: a multi-center study. Pan Afr Med J 23:1-8

6. Ekwunife O, Ugwu J, Okoli C, Modekwe V, Osuigwe A (2015) Parental circumcision preferences and early outcome of plastibell circumcision in a Nigerian tertiary hospital. Afr J Paediatr Surg 12(4):251

7. Neeto JMB, De Bessa JJ, Figueredo AA (2013) Prospective randomized trial comparing dissection with Plastibell circumcision. Int Braz J Urol 39(4):572-577

8. Moosa FA, Khan FW, Rao MH (2010) Comparison of complications of circumcision by 'Plastibell Device Technique' in male neonates and infants. J Pak Med Assoc 60(8):664-667

9. Al-Marhoon MS, Jaboub SM (2006) Plastibell circumcision: how safe is it?: experience at Sultan Qaboos University Hospital. Sultan Qaboos Univ Med J 6(1):17-20

10. Daniel Y (2007) Circumcision. In: Text Atlas of penile surgery. Informa Healthcare, p 27

11. Ofoha CG, Babangida SJ, Nabasu LE, Dakum NK (2018) Indications and safety of Plastibell circumcision in children. Int Surg J 5:1598-1601
12. Marwat AA, Hashmi ZA, Waheed D (2010) Circumcision with plastibell device : an experience with 780 children. Gomal J Med Sci 8(1):30-33

13. Netto JMB, de Araújo JG, de Almeida Noronha MF, Passos BR, Lopes HE, de Bessa J et al (2013) A prospective evaluation of Plastibell in older children. Int Br J Urol 39(4):558-564

14. Bode CO, Ikhesimojie S, Ademuyiwa AO (2010) Penile injuries from proximal migration of the Plastibell circumcision ring. J Ped Urol 6(1):23-27

15. Lazarus J, Alexander A, Rode H (2007) Circumcision complications associated with the Plastibell device. S Afr Med J 97(3):192-193

16. Palit V, Menebhi DK, Taylor I et al (2007) A unique service in UK delivering Plastibell circumcision: review of 9-year results. Pediatr Surg Int 23(1):45-48

17. Mak YLM (1995) Childhood circumcision: conventional dissection or plastibell device - a prospective randomized trial. Hong Kong Practitioner 17(3):101-105

18. Fraser IA, Allen MJ, Bagshaw PF, Johnstone M (1981) A randomized trial to assess childhood circumcision with the Plastibell device compared to a conventional dissection technique. Br J Surg 68(8):593-595

19. Van Howe RS (1997) Variability in penile appearance and penile findings: a prospective study. Br J Urol 80(5):776-782

20. Sidler D, Bode C, Desal A (2012) Circumcision. In: Paediatric surgery: a comprehensive text for Africa. GLOBAL HELP, p 554

\section{Publisher's Note}

Springer Nature remains neutral with regard to jurisdictional claims in published maps and institutional affiliations.

\section{Submit your manuscript to a SpringerOpen ${ }^{\circ}$ journal and benefit from:}

- Convenient online submission

- Rigorous peer review

- Open access: articles freely available online

- High visibility within the field

- Retaining the copyright to your article

Submit your next manuscript at springeropen.com 